\title{
On Placement and Efficient Resource Allocation of LAA/LTE-U Base Stations in HetNet
}

\author{
Anand M. Baswade*, Shashikiran M R ${ }^{\dagger}$, Bheemarjuna Reddy Tamma*, Antony Franklin A* \\ ${ }^{*}$ Indian Institute of Technology Hyderabad, India, ${ }^{\dagger}$ PES Institute of Technology Bengaluru, India. \\ Email: [cs14resch11002*, tbr*, antony.franklin*]@iith.ac.in, shashikiran.161996†@gmail.com
}

\begin{abstract}
LTE operation in unlicensed spectrum is considered as a promising solution to meet the increase in user data demand. Licensed Assisted Access (LAA), and duty cycled LTE-U are two options for LTE to operate in the unlicensed band for fair sharing of unlicensed spectrum with IEEE 802.11 (Wi-Fi). Due to restriction on the transmission power in the unlicensed band, LAA/LTE-U Base Stations (BSs) will get deployed mostly inside residential and office buildings to provide high data rates for indoor User Equipments (UEs). In an indoor scenario, walls and other obstacles in the communication path along with co-tier and cross-tier interferences decrease the Signal-to-Interference plus Noise Ratio (SINR) significantly which results in throughput decrease. Hence, an optimal placement of LAA BSs is essential. As the available bandwidth in the unlicensed spectrum is more compared to the licensed spectrum, an efficient resource allocation is also necessary for ensuring minimum throughput for the indoor UEs. In this paper, our goal is to find the optimal number of LAA/LTE-U BSs with minimum throughput guarantee inside the building using licensed and unlicensed bands. To do this, we formulate an optimization model (MinLAA) for LAA BSs placement which is Mixed Integer Non-Linear Programming (MINLP) problem. So, we propose a heuristic algorithm to find the minimum number of LAA/LTE-U BSs such that all the users inside the building get minimum guaranteed throughput.
\end{abstract}

\section{INTRODUCTION}

The recent increase in data traffic due to increases in usage of smartphones and tablets has increased the demand for spectrum. The wireless traffic is expected to expand further in coming years [1]. Users consume more data in indoor scenarios with rich content including the video traffic [2]. The mobile operator can boost the datarate for outdoor users using Macro BS, but they are unable to increase the data rates for indoor users. Due to high penetration loss through walls and floors, the indoor users suffer from low signal strength. As indoor users consume more data, the low Signalto-Noise Ratio (SNR) in the indoor regions is a very important problem to solve. This problem is addressed in literature with the help of low power node a.k.a. Femto cell inside the building so that SNR in indoor regions can be boosted. Hence, Femto placement inside the building is necessary to meet the indoor user demand. An arbitrary placement of Femto Base Stations (BSs) is not efficient as it may reduce the spectrum efficiency due to co-tier interference. Hence, to increase the spectrum efficiency and to boost SINR to a great extent, optimal placement of Femto BSs is necessary.

Further, the recent increase in the demand from indoor users with limited licensed spectrum forces mobile operators to find alternate solutions. The use of unlicensed spectrum [3],
[4] is considered as one of the promising solution to meet such high demand from indoor users. LTE in unlicensed [3], [4] uses carrier aggregation feature of LTE-Advance where it aggregates licensed component carrier with the unlicensed component carrier. The licensed carrier acts as the primary cell where along with data the control informations are transferred whereas the unlicensed carrier acts as a secondary cell where only data traffic is transmitted. The operating frequency of LTE in unlicensed spectrum is in $5 \mathrm{GHz}$ which is high than the operating frequency of licensed spectrum $(400 \mathrm{MHz}-$ $2.6 \mathrm{GHz}$ ), hence more wall and floor losses inside the building for unlicensed spectrum compared to licensed spectrum. This results in different coverage regions and SINR values of licensed and unlicensed spectrum. Hence, efficient allocation of licensed and unlicensed spectrum for indoor users is necessary for better spectral efficiency. Further, the presence of Wi-Fi Access Points (APs) makes the problem more challenging as LAA BSs share the unlicensed channel with Wi-Fi when it is in energy detection region. An energy detection region is a region in which LAA BS receives Wi-Fi signal energy more than its Energy Detection Threshold (EDT).

In this paper, we focus on efficient resource allocation (i.e., licensed and unlicensed spectrum) in the presence of Wi-Fi AP to meet minimum throughput demand of each indoor user with the minimum number of LAA/LTE-U BSs inside the building.

\section{RELATED WORK}

Recently, the use of LTE operation in the unlicensed spectrum has received a lot of attention in the research community, with a major issue of fair sharing of unlicensed spectrum with other radio access technologies like Wi-Fi. In [5], the authors discussed the benefits and challenges of LTE in unlicensed spectrum. Most of the work in the literature focus on fair coexistence of LTE-U/LAA and Wi-Fi [6], [7]. But, to the best of our knowledge, the placement of LAA/LTE-U has not received much attention till date. Random placement of LAA/LTE-U nodes inside the buildings can create a lot of issues like co-tier interference, frequent handover. Also, it may not guarantee the throughput requirements in all the regions of the building. An optimal placement of the LAA/LTE-U can solve the problems mentioned above and gives better spectral efficiency. In literature, several optimal LTE Femto placement approaches have been proposed, taking into account different parameters such as building dimension, floors, walls, etc. In [8], [9], a single Femto is placed in a multi-room indoor 
environment based on the location of the Macro BS. In [10], authors considered an indoor building scenario for placement of LTE Femtos. But, due to the absence of unlicensed spectrum the solution is not directly applicable to LAA/LTE-U BSs as the presence of Wi-Fi can change placement. As well as our goal is to achieve minimum throughput for each users which also depends on available bandwidth, and in the unlicensed spectrum we have more bandwidth compared to licensed spectrum. Existing works which are based only on the licensed spectrum for placement cannot be applied directly to LAA/LTE-U BSs because of both licensed and unlicensed spectrum. Hence, in this paper, we considered LTE-U/LAA BSs placement problem with minimum throughput guarantee for indoor users.

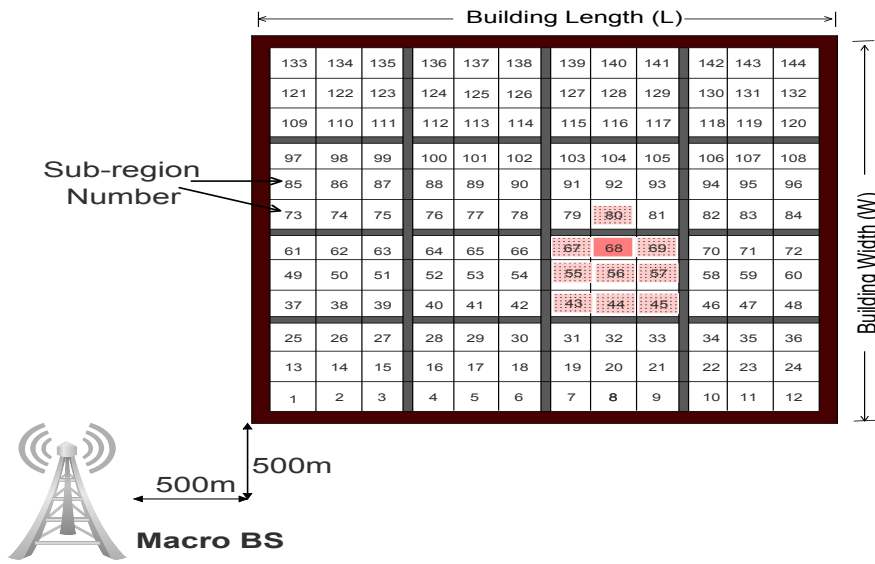

Fig. 1. System model with a building having 16 rooms and a Macro BS outside the building along with one Wi-Fi AP at sub-region 68 with its energy detection region.

\section{System MOdEL}

\section{A. Building Model}

Our system model is shown in Fig. 1 where the Macro base station is $500 \mathrm{~m}$ away from the first sub-region in horizontal and vertical directions of the building. The building has length $L$, width $W$, and height $H$. The building is divided into rooms where each room has a set of sub-regions, with each subregion of has length $l$, width $w$, and height $H$. Fig. 1 shows the considered building scenario. The thick lines represent walls of the rooms, and inner squares represent sub-regions. We assume that the SINR inside each sub-region is constant as the dimension of sub-regions are smaller compared to the entire building. We consider single licensed and unlicensed frequency band (i.e., frequency reuse one). We also consider that a Wi-Fi AP is present inside the building, Fig. 1 shows Wi-Fi AP position along with its energy detection region.

\section{B. Channel Model}

As LAA BS operates in both licensed and unlicensed spectrum, we use two different path loss models for licensed and unlicensed spectrum. The path loss (in $\mathrm{dB}$ ) between LAA BS transmitting in licensed spectrum and the sub-regions in the building is given by [11] as follows:

$$
P L_{\text {Femto }}^{l}=37+30 \log _{10} d+N \sigma^{l}+18.3 v^{\frac{v+2}{v+1}-0.46}
$$

The path loss in $\mathrm{dB}$ between LAA BS transmitting in unlicensed spectrum and the sub-regions in the building is:

$$
P L_{\text {Femto }}^{u}=20 \log _{10} f+30 \log _{10} d+N \sigma^{u}-28
$$

where $f$ is the transmission frequency of unlicensed spectrum in $\mathrm{MHz}, d$ is the distance between LAA BS and each subregion in meters, $N$ is the number of walls between the LAA BS and the sub-region, $\sigma^{u}$ and $\sigma^{l}$ are wall losses of unlicensed and licensed spectrum in $\mathrm{dB}$, respectively and $v$ is the total number of floors in the building.

The path loss (in $\mathrm{dB}$ ) between Macro BS located outside the building and the indoor sub-regions of the building [11] is:

$$
P L_{\text {Macro }}=49+40 \log _{10} \frac{D}{1000}+30 \log _{10} F+K \sigma^{l}
$$

where $D$ is the distance between each sub-region and the Macro BS in meters, $F$ is the transmission frequency of Macro BS in $\mathrm{MHz}$ and $K$ is the number of walls between the Maro $\mathrm{BS}$ and the sub-regions.

The path loss between Wi-Fi and the sub-regions remains the same as path loss model of LAA BS transmitting in the unlicensed spectrum ( $\left.P L_{\text {Femto }}^{u}\right)$ as given in Eqn. (2).

\begin{tabular}{|c|c|}
\hline Notation & Definition \\
\hline $\bar{I}$ & Set of all indoor sub-regions \\
\hline$C$ & Set of all sub-regions where $\mathrm{WiFi} \mathrm{AP}$ is located \\
\hline$b_{i}$ & 1 if LAA BS is placed at sub-region $i$, otherwise 0 \\
\hline$a_{j i}^{l}$ & $\begin{array}{l}1 \text { if } j^{t h} \text { sub-region of the building is associated with the } \\
\text { licensed spectrum of BS located at sub-region } i \text {, otherwise } 0\end{array}$ \\
\hline$\overline{a_{j i}^{u}}$ & $\begin{array}{l}1 \text { if } j^{t h} \text { sub-region of the building is associated with the } \\
\text { unlicensed spectrum of BS located at sub-region } i \text {, otherwise } 0\end{array}$ \\
\hline$G_{j i}^{u}$ & Unlicensed channel gain to sub-region $j$ from LAA BS at $i$ \\
\hline$G_{j i}^{l}$ & Licensed channel gain to sub-region $j$ from LAA BS at $i$ \\
\hline$G_{j c}^{w}$ & Unlicensed channel gain to sub-region $j$ from Wi-Fi AP at $c$ \\
\hline$P_{\max }^{u}$ & Maximum Tx power of unlicensed spectrum from LAA BS \\
\hline$P_{\max }^{l}$ & Maximum Tx power in licensed spectrum from LAA BS \\
\hline$P^{w}$ & Tx power of Wi-Fi AP \\
\hline$P^{m}$ & Tx power of licensed spectrum from Macro BS \\
\hline$N$ & System noise \\
\hline$S I N R_{t h}^{u}$ & $\begin{array}{l}\text { Minimum SINR required in unlicensed spectrum for a sub- } \\
\text { region from LAA BS for communication with lowest rate }\end{array}$ \\
\hline$S I N R_{t h}^{l}$ & $\begin{array}{l}\text { Minimum SINR required in licensed spectrum for a sub-region } \\
\text { from LAA BS for communication with lowest rate }\end{array}$ \\
\hline$S I N R_{j}^{l}$ & SINR in licensed spectrum at sub-region $j$ \\
\hline$S I N R_{j}^{u}$ & SINR in unlicensed spectrum at sub-region $j$ \\
\hline$R_{\min }$ & Minimum throughput required for each sub-region (in Mbps) \\
\hline$B W_{j i}^{l}$ & $\begin{array}{l}\text { Licensed bandwidth allocated to a sub-region } j \text { which is } \\
\text { associated with LAA BS at sub-region } i\end{array}$ \\
\hline$B W_{j i}^{u}$ & $\begin{array}{l}\text { Unlicensed bandwidth allocated to a sub-region } j \text { which is } \\
\text { associated with LAA BS at sub-region } i\end{array}$ \\
\hline$T B W_{i}^{l}$ & Total licensed bandwidth of BS located at sub-region $i$ \\
\hline$\frac{2}{T B W_{i}^{u}}$ & Total unlicensed bandwidth of BS located at sub-region $i$ \\
\hline$u_{j}$ & $\begin{array}{l}1 \text { if jth sub-region is outside energy detection region of all the } \\
\text { Wi-Fi APs in the building, otherwise } 0\end{array}$ \\
\hline$v_{j c}$ & $\begin{array}{l}0 \text { if jth sub-region is inside energy detection region of the } \\
\text { Wi-Fi AP located at sub-region } c \text {, otherwise } 1\end{array}$ \\
\hline$O P T_{P o s}$ & Optimal positions of LAA BSs returned by MinBS Algorithm \\
\hline$E D T_{c}$ & $\begin{array}{l}\text { Set of all sub-regions inside energy detection region of Wi-Fi } \\
\text { located in sub-region } c\end{array}$ \\
\hline
\end{tabular}

TABLE I

GLOSSARY 


\section{PROPOSED WORK}

\section{A. Aim}

Since most of the high data rate users are the indoor users, the objective of our work is to provide uniform throughput to each sub-region in the indoor area, assuming the uniform distribution of users in each sub-region.

\section{B. Formulation}

The optimization model we have designed is called as MinLAA (Minimum number of LAA BSs) model, and the parameters considered in our formulation are represented in Table I. The objective of the formulation is to minimize the number of LAA BSs as given below.

$$
\min \sum_{i \in I} b_{i}
$$

To minimize the number of LAA BSs required, we have to utilize the resources to the maximum extent possible. Hence, we transmit the licensed and unlicensed spectrum at their maximum powers. To achieve our goal, the first thing we need to know is the $S I N R^{l}$ and $S I N R^{u}$ in each sub-region.

$$
S I N R_{j}^{l}=\frac{G_{j i}^{l} P_{\max }^{l} a_{j i}^{l}}{N+\sum_{k \in I \backslash i} G_{j k}^{l} P_{\max }^{l} b_{k}+\sum_{m \in M} G_{j m}^{m} P^{m}} \quad \forall j, i \in I
$$

Eqn. (5) is used to find the $S I N R^{l}$ in each sub-region. The numerator consists of the received signal from the LAA $\mathrm{BS}$ at $i$ to which the considered sub-region $j$ is associated. The denominator consists of the sum of system noise and interference from other LAA BSs and the Macro BSs located outside the indoor area.

$$
S I N R_{j}^{u}=\frac{G_{j i}^{u} P_{\max }^{u} a_{j i}^{u}}{N+\sum_{k \in I \backslash i} G_{j k}^{u} P_{\max }^{u} b_{k} u_{k}+\sum_{c \in C} G_{j c}^{w} P^{w} v_{i c}} \quad \forall j, i \in I
$$

To find $S I N R^{u}$ in each sub-region, we use Eqn (6). The numerator consists the received signal strength of unlicensed spectrum for the considered sub-region $j$ from its associated LAA BS located at $i$. The denominator consists of the sum of system noise, and interference from other LAA BSs and the Wi-Fi APs. For a given sub-region $j$ there may be many other Wi-Fi APs and LAA BSs in the building other the one to which it is associated, and interference from them has to be considered to find $S I N R_{j}^{u}$. But, when both LAA BS and Wi-Fi AP are inside the energy detection region then both shares the channel. In that case, interference to sub-region $j$ is either from LAA BS or Wi-Fi AP at any given time but not from both. When LAA BS is inside energy detection region of Wi-Fi AP either of them will be transmitting as explained above and the amount of interference they cause to a subregion $j$ is almost the same because of similar properties of LAA BS in unlicensed spectrum and Wi-Fi AP. Hence, to simplify our formulation, interference from those LAA $\mathrm{BS}$ which are inside energy detection region of Wi-Fi AP is replaced by interference from that $\mathrm{Wi}-\mathrm{Fi} \mathrm{AP}$ itself i.e., interference from Wi-Fi AP is considered to be existing for full duration instead of interference from LAA BS for some duration and Wi-Fi AP for some duration. The term $u_{k}$ is used to consider the interference from LAA BS which are completely outside energy detection region of all sub-regions $\left(u_{k}=1\right)$. If it is inside energy detection region of a $\mathrm{Wi}-\mathrm{Fi}$ AP located at $c$ then interference from that Wi-Fi AP is added since $u_{k}=0$. If the LAA BS to which the considered subregion $j$ is associated lies inside the energy detection region of a Wi-Fi AP at $c$, then interference from this Wi-Fi AP will not exist for $j$ as they share the same channel. Hence, $v_{i c}=0$ only if the associated LAA BS is inside energy detection region of $\mathrm{Wi}-\mathrm{Fi} \mathrm{AP}$ at $c$, in rest all the cases Wi-Fi interference is added.

Before associating a sub-region to a particular LAA BS, it has to be verified that minimum SINR threshold (i.e., minimum SINR to have a communication with lowest rate) has to be satisfied. It is to be noted that for the sub-regions which are allocated with unlicensed spectrum, SINR threshold has to be satisfied by both licensed and unlicensed spectrum as well. It is because the control signals for the use of unlicensed spectrum are to be sent only through licensed spectrum. Hence, the unlicensed spectrum cannot be used until and unless control signals through licensed spectrum are sent.

$$
\begin{aligned}
& \text { Inf*(1- } \left.a_{j i}^{l}\right)+S I N R_{j}^{l} b_{i} \geq S I N R_{t h}^{l} \quad \forall j, i \in I \\
& \text { Inf*(1-a } \left.a_{j i}^{u}\right)+S I N R_{j}^{l} b_{i} \geq S I N R_{t h}^{l} \quad \forall j, i \in I
\end{aligned}
$$

Eqns. (7) and (8) ensure that SINR in licensed spectrum for all sub-regions exceed $S I N R_{t h}^{l}$. If a sub-region $j$ is associated with licensed spectrum from LAA BS at $i$, then $a_{j i}^{l}$ is 1 and it checks for $S I N R_{t h}^{l}$, otherwise $a_{j i}^{l}$ is 0 and the equation is simply neglected as $\operatorname{Inf}$ is very large value. If a sub-region is associated with unlicensed spectrum from LAA BS at $i$, then $a_{j i}^{u}$ is 1 and it checks for $S I N R_{t h}^{l}$, otherwise $a_{j i}^{u}$ is 0 and the equation is simply neglected due to $\operatorname{Inf}$.

$$
\text { Inf } *\left(1-a_{j i}^{u}\right)+S I N R_{j}^{u} b_{i} \geq S I N R_{t h}^{u} \quad \forall j, i \in I
$$

Eqn. (9) ensures that for the sub-regions allocated with unlicensed spectrum, $S I N R_{t h}^{u}$ is satisfied or not. If a sub-region is associated with unlicensed spectrum from LAA BS at $i$, then $a_{j i}^{u}$ is 1 and it checks for $S I N R_{t h}^{u}$, otherwise $a_{j i}^{u}$ is 0 and the equation is simply neglected due to $\operatorname{In} f$.

$$
\sum_{j \in I} a_{j i}^{l}+a_{j i}^{u}=1 \quad \forall i \in I
$$

For maintaining uniform throughput for all sub-regions and to minimize the number of LAA BSs at the same time, either licensed or unlicensed spectrum has to be allocated for each sub-region. The bandwidth allocation to achieve minimum throughput is based on Shanon's Capacity theorem.

$$
B W_{j i}^{l}=\frac{a_{j i}^{l} R_{m i n}}{\log _{2}\left(1+S I N R_{j}^{l}\right)} \quad \forall j, i \in I
$$


For a sub-region $j$ associated with licensed spectrum from LAA BS at $i, a_{j i}^{l}$ is 1 and the bandwidth to be allocated is calculated using Eqn. (11).

$$
B W_{j i}^{u}=\frac{a_{j i}^{u} R_{\min }}{\log _{2}\left(1+S I N R_{j}^{u}\right)}\left(\left(1-u_{i}\right)+1\right) \quad \forall j, i \in I
$$

For a sub-region $j$ associated with unlicensed spectrum from LAA BS at $i, a_{j i}^{u}$ is 1 and the bandwidth to be allocated is calculated using Eqn. (12). If the LAA BS is sharing channel with any Wi-Fi AP, then double the bandwidth has to be needed for maintaining uniform throughput for the sub-region. The term $u_{i}$ ensures this bandwidth allocation as necessary.

$$
\sum_{i \in I} \sum_{j \in I} B W_{j i}^{u} a_{j i}^{u} \leq T B W_{i}^{u}
$$

The bandwidth allocation has to be in a way that it should not exceed the maximum available bandwidth of each LAA BS. Eqn. (13) ensures that the total allocated unlicensed bandwidth from each LAA BS does not exceed the total available bandwidth of that LAA BS.

$$
\sum_{i \in I} \sum_{j \in I} B W_{j i}^{l} a_{j i}^{l} \leq T B W_{i}^{l}
$$

Eqn. (14) ensures that the total allocated licensed bandwidth from each LAA BS does not exceed the total available bandwidth of that LAA BS.

Hence, our objective equation for MinLAA model is (4) subject to constraints given in Eqns. (7), (8), (9), (10), (13), and (14). The formulation for MinLAA model discussed is Mixed Integer Non Linear Programming (MINLP) Problem which cannot be easily solved using traditional MINLP algorithm, hence we designed our heuristic algorithm and tested for different scenarios and obtained enough simulation results to prove the reliability of the algorithm.

\section{Heuristic Approach for MinLAA Model}

We propose MinBS algorithm to find the minimum number of LAA BSs such that each sub-region inside building gets minimum throughput.

1) MinBS Algorithm: In the MinBS Algorithm 1, the Combinations $(\mathrm{x}, \mathrm{y})$ function returns all the possible combinations of set $x$ with length of each combination is of size $y$, and Associativity $(\mathrm{x}, \mathrm{y})$ function associates the sub-regions in the set $y$ to the optimal LAA BS among the available set of LAA BSs located in sub-regions given by the set $x$. To reduce the complexity of the algorithm, we only consider center subregion of each room. If the room center can not get $R_{\text {min }}$, then other sub-regions inside that room might not get $R_{\min }$ and hence the combinations of LAA BS which does not satisfy room centers can be eliminated instead of checking for each sub-region. Instead of checking for all sub-regions in the building from first, we select only those combinations of LAA BSs which serve room-centers. Hence, we start by considering only center sub-region of each room (from line 3 ). Place LAA BS in the center sub-region and find associativity

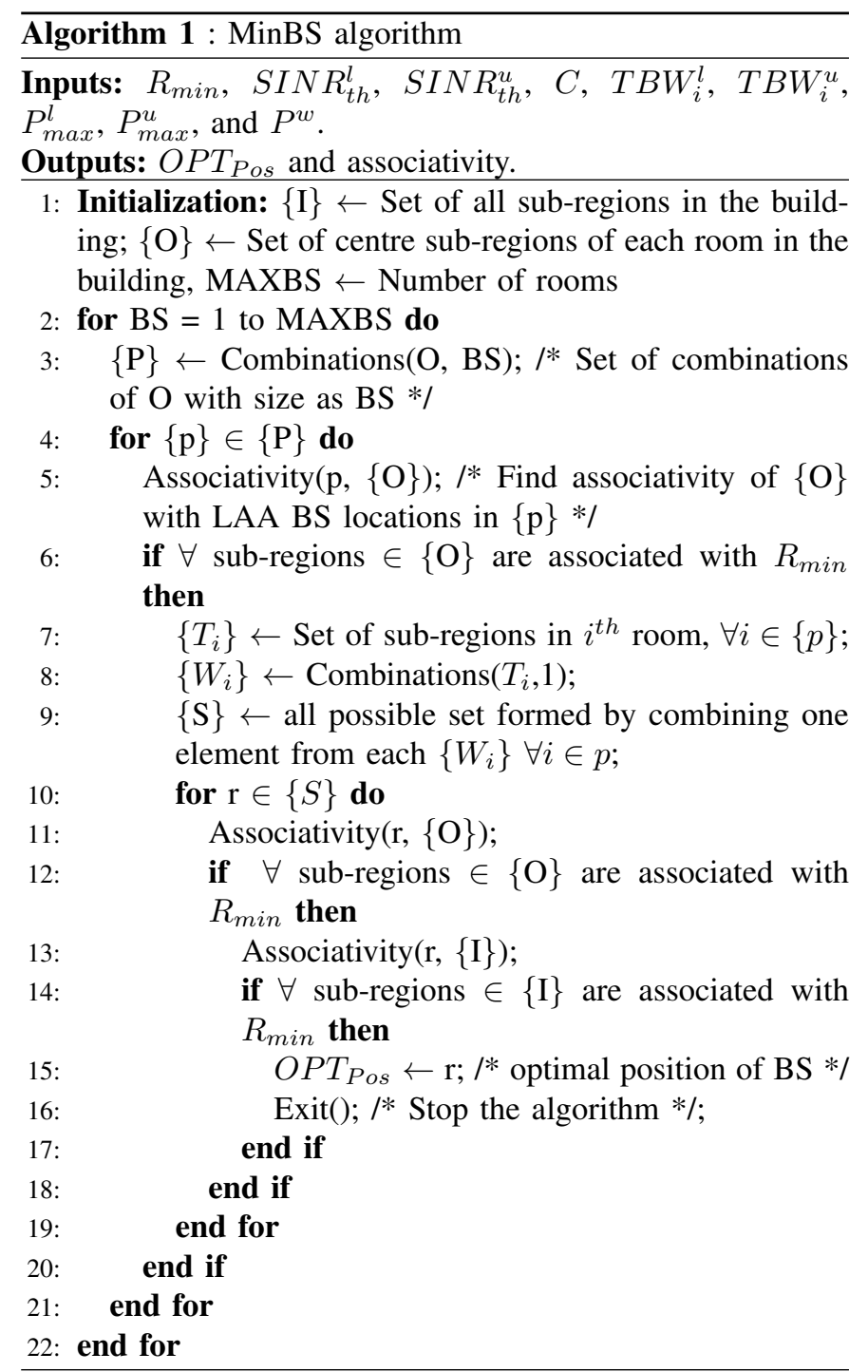

(line 5) using Algorithm 2 such that the center sub-region of all the rooms is getting $R_{\min }$ (line 6). Once we find the room-centers where LAA BSs are located, we extend the set of possible solutions by placing LAA BS in each sub-region in the room in which it is present (from lines 7 to 9). In the set of possible solutions (line 10), only for those solutions which satisfy all room-centers, we check whether each sub-region in the building (in lines 13 and 14) is getting $R_{\text {min }}$ by this positioning of LAA/LTE-U BSs.

2) Associativity Algorithm: The Associativity Algorithm 2 efficiently associates a group of sub-regions to BSs based on SINR and available bandwidth of licensed and unlicensed spectgrum. From the set of given sub-regions and locations of LAA BS (line 1), start with first sub-region $j$ (line 2). From the available LAA BS, find the one which gives maximum licensed SINR (from lines 4 to 7). Check whether $S I N R_{j m}^{l}$ is above $S I N R_{t h}^{l}$ (line 8). Now compare the licensed SINR with Unlicensed SINR and associate to the one which is better by verifying whether bandwidth is available or not (from lines 9 
to 17) and deduct the bandwidth allocated from the total available bandwidth. Repeat the steps for all the sub-regions.

The proposed MinBS algorithm with the help of Associativity() finds out the minimum number of LAA BSs required along with positions in such a way that each sub-region gets minimum guaranteed throughput.

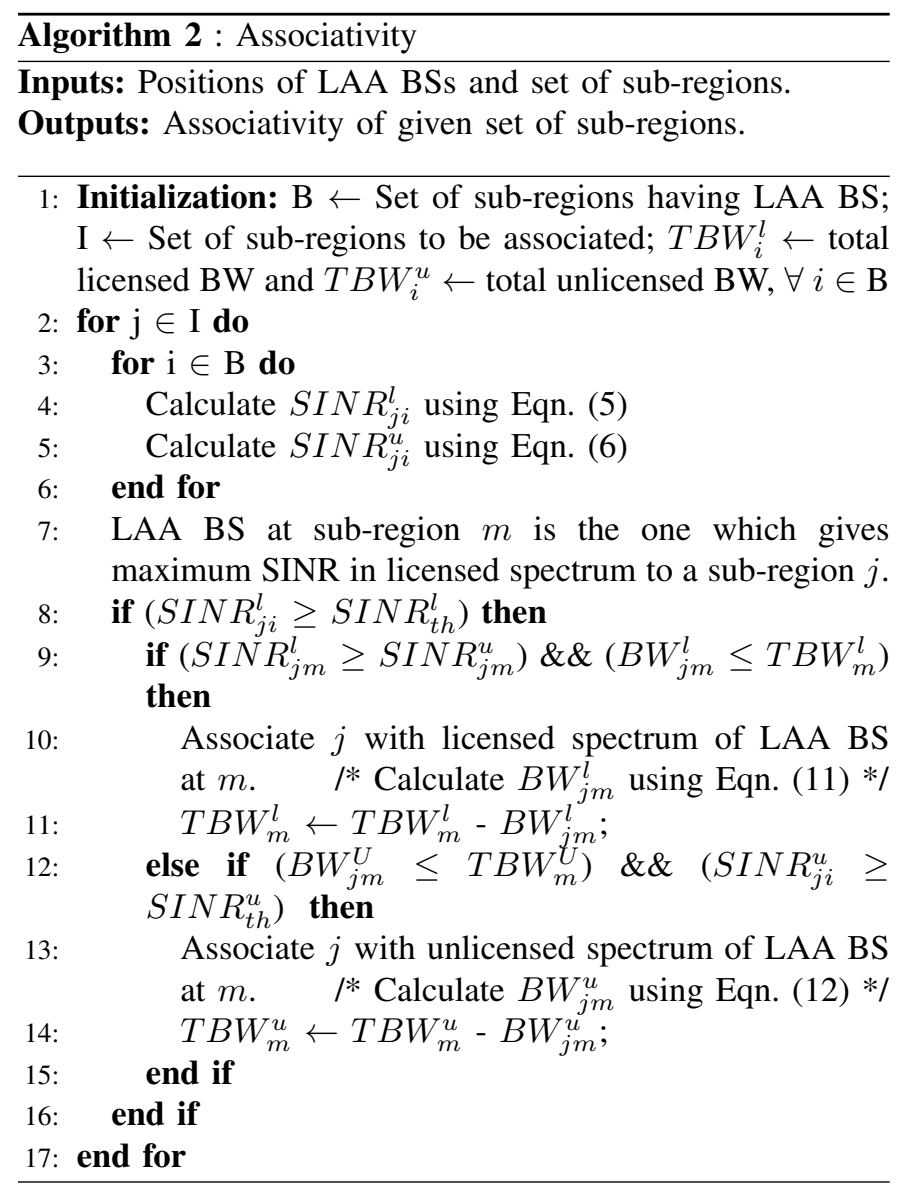

\section{EXPERIMENTAl SETUP AND Numerical Results}

The system model described in Section III is simulated in MATLAB using simulation parameters as shown in Table II. We placed a Wi-Fi AP inside the building at sub-region

TABLE II

SIMULATION PARAMETERS

\begin{tabular}{|l|l|}
\hline \multicolumn{1}{|c|}{ Parameter } & \multicolumn{1}{c|}{ Value } \\
\hline \hline Building dimensions & $48 \mathrm{~m} \times 48 \mathrm{~m} \times 3 \mathrm{~m}$ \\
\hline Number of rooms & 16 \\
\hline Room dimensions & $12 \mathrm{~m} \times 12 \mathrm{~m} \times 3 \mathrm{~m}$ \\
\hline Number of inner Sub-regions & 144 \\
\hline Sub-region dimension & $4 \mathrm{~m} \times 4 \mathrm{~m} \times 3 \mathrm{~m}$ \\
\hline$S I N R_{T h}^{l}, S I N R_{T h}^{u}$ & $-2 \mathrm{~dB}$ \\
\hline$R_{\min }$ & $2 \mathrm{Mbps}$ \\
\hline Wall loss (in Licensed) & $10 \mathrm{~dB}$ \\
\hline Wall loss (in Unlicensed) & $13 \mathrm{~dB}$ \\
\hline Macro transmit power $\left(P^{m}\right)$ & $46 \mathrm{dBm}(39.8 \mathrm{~W})$ \\
\hline$P_{\max }^{l}, P_{\max }^{u}$ & $20 \mathrm{dBm}(0.1 \mathrm{~W})$ \\
\hline$T B W_{i}^{l}, T B W_{i}^{l}$ & $20 \mathrm{MHz}$ \\
\hline$P^{w}$ & $20 \mathrm{dBm}(0.1 \mathrm{~W})$ \\
\hline Macro BS height & $35 \mathrm{~m}$ \\
\hline
\end{tabular}

68, and the energy detection region of AP is as shown in Fig. 1. After running the MinBS algorithm for $R_{\min }$ (i.e., minimum throughput required in each sub-region) of $2 \mathrm{Mbps}$, the optimal number of LAA BSs required are two and the obtained positions of BSs are at sub-regions 3, and 117. The SINR distribution in licensed and unlicensed spectrum for all the sub-regions with the obtained placement are shown in Figs. 2 and 3, respectively. In figure, $\mathrm{X}$ and $\mathrm{Y}$ axes indicate sub-region number whereas $\mathrm{Z}$ axis indicates SINR values. From Fig. 3, it is clear that the sub-regions around Wi-Fi AP have very poor SINR values in unlicensed spectrum. After allocating bandwidth obtained using our algorithm, the SINR of allocated spectrum of each sub-region is given in Fig. 4. In our approach, we have started allocation of spectrum from sub-region 1. Fig. 5 shows the associativity of each sub-region. It is observed that rooms R1 to R6 are associated with the licensed spectrum of BS L1 because in all these rooms SINR in licensed spectrum from BS L1 is better compared to BS L2. Further, SINR in licensed is better compared to SINR in unlicensed spectrum with enough bandwidth of licensed spectrum from BS L1. It can be observed that for room R9, even though SINR in licensed from BS L1 is better than SINR in unlicensed, there is not enough bandwidth available in the licensed spectrum and hence all sub-regions in room R9 are getting $R_{\text {min }}$ from the unlicensed spectrum of BS L1.

The same pattern is observed for BS L2 as well. Until the licensed spectrum of BS L2 is available, the sub-regions having better SINR in licensed than the SINR in unlicensed get associated with licensed spectrum, after which even though SINR in licensed is better than SINR in unlicensed, the subregions get associated with unlicensed spectrum. As the Wi-Fi AP present in room R7 (at sub-region 68) and LAA BS is outside energy detection region hence, both can transmit at the same time. The simultaneous transmissions of AP and BS in unlicensed, decreases the SINR in unlicensed spectrum whereas SINR in licensed spectrum is still good hence, that region is associated with licensed spectrum of BS L2. Fig. 6 shows the CDF for SINR in licensed, SINR in unlicensed, and combined SINR (combined SINR is SINR of the spectrum whose $\mathrm{BW}$ is allocated). It can be observed that $\mathrm{CDF}$ for combined SINR is better compared to other two. The better CDF of SINR leads to efficient bandwidth allocation for maintaining $R_{\min }$ in each sub-region which in turn minimizes the number of LAA BSs required.

By varying $R_{\min }$ :- To study the reliability of our heuristic approach and validate the results, we have considered a scenario by varying the $R_{\min }$ requirement for the building. By varying $R_{\text {min }}$ from $1 \mathrm{Mbps}$ to $5 \mathrm{Mbps}$ in steps of $1 \mathrm{Mbps}$, it can be observed that until $3 \mathrm{Mbps}$, the building can be served by two LAA BS. This was achieved by efficient resource allocation i.e., by placing the LAA BSs at optimal positions and by allocating licensed and unlicensed spectrum efficiently. For $R_{\min } 4 \mathrm{Mbps}$ and $5 \mathrm{Mbps}$, three LAA BSs are needed to serve the building. Hence, these results are in accordance with the theoretical assumptions because with an increase in $R_{\min }$ the number of LAA BSs required should either increase 


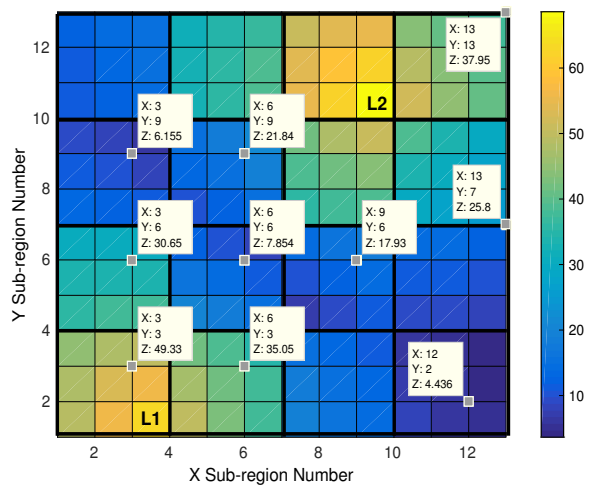

Fig. 2. SINR (in $\mathrm{dB}$ ) distribution in licensed spectrum inside the building.

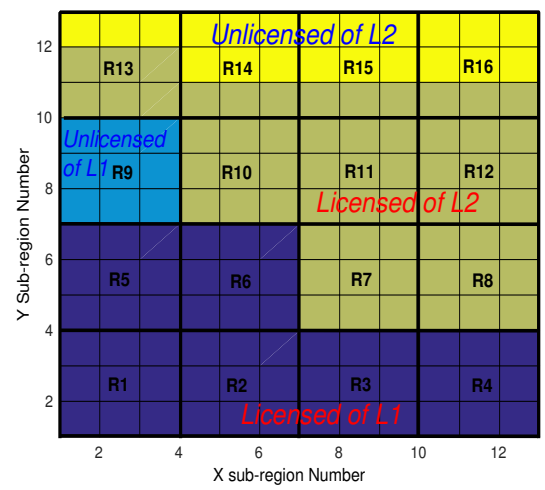

Fig. 5. Associativity of each sub-region along with licensed and unlicensed allocation.

or remain the same.

By varying number of APs:- To study the variation in the number of BSs required by varying the number of Wi-Fi APs in the building, we varied Wi-Fi APs from one to five in the building. The simulation results of the considered scenario are shown in Table III. From one Wi-Fi AP till three Wi-Fi APs, only two LAA BSs are required to serve each sub-region with $R_{\text {min }}$. The coordinates of Wi-Fi APs along with the positions of required LAA BSs are also shown. When there are four or five Wi-Fi APs in the building, the number of LAA BSs required increases by one. Hence, the trend in variation of simulation results for different scenarios has been studied, and the variation of results by varying parameters validate our heuristic approach as the results are as expected in reality.

TABLE III

RESULTS WITH VARYING NUMBER OF WI-FI APS

\begin{tabular}{|c|l|c|l|}
\hline $\begin{array}{l}\text { No. of } \\
\text { APs }\end{array}$ & $\begin{array}{l}\text { AP Positions } \\
\text { (sub-region No.) }\end{array}$ & $\begin{array}{l}\text { Required } \\
\text { No. of BSs }\end{array}$ & $\begin{array}{l}\text { BS Positions } \\
\text { (sub-region No.) }\end{array}$ \\
\hline \hline 1 & 68 & 2 & 3,117 \\
\hline 2 & 68,113 & 2 & 64,68 \\
\hline 3 & $26,68,113$ & 2 & 43,138 \\
\hline 4 & $26,68,113,119$ & 3 & $25,30,120$ \\
\hline 5 & $26,68,74,113,119$ & 3 & $2,32,116$ \\
\hline
\end{tabular}

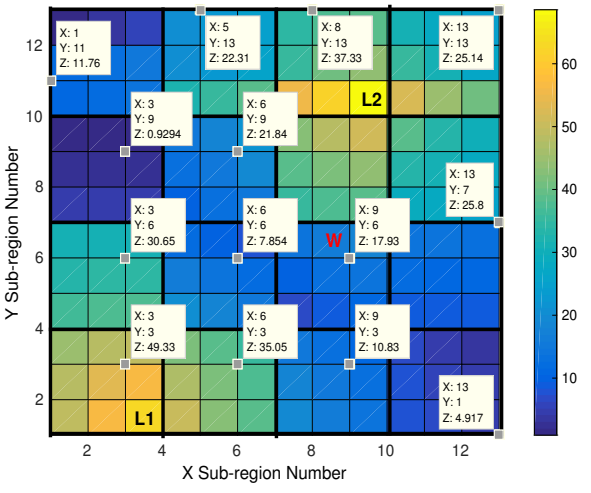

Fig. 4. Combined SINR (in $\mathrm{dB}$ ) distribution (SINR of allocated spectrum) in each sub-region.

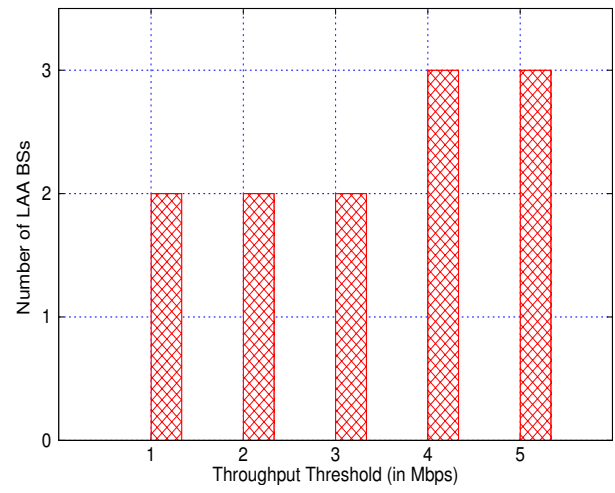

Fig. 7. Number of LAA BSs required with varying $R_{\min }$

\section{CONCLUSIONS}

In this paper, to guarantee minimum throughput for each user either using licensed and unlicensed spectrum with minimum number of LAA/LTE-U BSs, we formulated an optimal LAA/LTE-U BS placement problem. Based on SINR in licensed and unlicensed spectrum, we allocated bandwidth to each sub-region in such a way that minimum throughput of each sub-region is satisfied with minimum number of LAA/LTE-U BSs. The efficiency of proposed algorithm is shown with sufficient simulation study.

\section{REFERENCES}

[1] "Cisco Visual Networking Index: global mobile data traffic forecast update, 2016-2021." Cisco White Paper, Feb 2017.

[2] "Ericsson mobility report." Ericsson, June 2017.

[3] 3GPP, "TSGRAN; Study on Licensed-Assisted Access to Unlicensed Spectrum,” Tech. Rep. TR 36.889 V13.0.0, June 2015.

[4] "LTE in Unlicensed Spectrum: Harmonious Coexistence with Wi-Fi," Qualcomm White Paper, June 2014.

[5] R. Zhang, M. Wang, et al., "LTE-Unlicensed: the future of spectrum aggregation for cellular networks," IEEE Wireless Communications, vol. 22 , no. 3 , pp. $150-159,2015$.

[6] A. Babaei, J. Andreoli-Fang, et al., "On the impact of LTE-U on Wi-Fi performance," International Journal of Wireless Information Networks, vol. 22, no. 4, pp. 336-344, 2015.

[7] "LTE-U Technical Report." LTE-U Forum [Online]. Available: http:// www.lteuforum.org/documents.html, 2015. 
[8] W. Guo, S. Wang, et al., "Automated small-cell deployment for heterogeneous cellular networks," Communications Magazine, IEEE, vol. 51, no. 5, pp. 46-53, 2013.

[9] W. Guo and S. Wang, "Interference-aware self-deploying femto-cell," IEEE wireless communications letters, vol. 1, no. 6, pp. 609-612, 2012.

[10] V. Sathya et al., "On placement and dynamic power control of femtocells in LTE hetnets," in Global Communications Conference (GLOBECOM), 2014 IEEE, pp. 4394-4399, IEEE, 2014.

[11] K. Han, Y. Choi, et al., "Optimization of femtocell network configuration under interference constraints," in WiOPT 2009., IEEE, 2009. 